\title{
Temperature Dependence of Laser-Induced Demagnetization in Ni: A Key for Identifying the Underlying Mechanism
}

\author{
T. Roth, ${ }^{1}$ A. J. Schellekens, ${ }^{2}$ S. Alebrand, ${ }^{1}$ O. Schmitt, ${ }^{1}$ D. Steil,,${ }^{1}$ B. Koopmans, ${ }^{2}$ M. Cinchetti, ${ }^{1 *}$ and M. Aeschlimann ${ }^{1}$ \\ ${ }^{1}$ Department of Physics and Research Center OPTIMAS, University of Kaiserslautern, \\ Erwin-Schrödinger-Strasse 46, 67663 Kaiserslautern, Germany \\ ${ }^{2}$ Department of Applied Physics, Center for NanoMaterials (cNM), Eindhoven University of Technology, \\ P.O. Box 513, 5600 MB Eindhoven, The Netherlands
} (Received 27 January 2011; published 16 May 2012)

\begin{abstract}
The microscopic mechanisms responsible for the ultrafast loss of magnetic order triggered in ferromagnetic metals by optical excitation are still under debate. One of the ongoing controversies is about the thermal origin of ultrafast demagnetization. Although different theoretical investigations support a main driving mechanism of thermal origin, alternative descriptions in terms of coherent interaction between the laser and the spin system or superdiffusive spin transport have been proposed. Another important matter of debate originates from the experimental observation of two time scales in the demagnetization dynamics of the $4 f$ ferromagnet gadolinium. Here, it is still unclear whether it is necessary to invoke two distinct microscopic mechanisms to explain such behavior, or if one single mechanism is indeed sufficient. To uncover the physics behind these two unsolved issues, we explore the dependence of ultrafast-demagnetization dynamics in nickel through a survey of different laser intensities and ambient temperatures. Measurements in a large range of these external parameters are performed by means of the time-resolved magneto-optical Kerr effect and display a pronounced change in the maximum loss of magnetization and in the temporal profile of the demagnetization traces. The most striking observation is that the same material system (nickel) can show a transition from a one-step (one time scale) to a two-step (two time scales) demagnetization, occurring on increasing the ambient temperature. We find that the fluence and the temperature dependence of ultrafast demagnetization-including the transition from one-step to two-step dynamics - are reproduced theoretically assuming only a single scattering mechanism coupling the spin system to the temperature of the electronic system. This finding means that the origin of ultrafast demagnetization is thermal and that only a single microscopic channel is sufficient to describe magnetization dynamics in the $3 d$ ferromagnets on all time scales.
\end{abstract}

DOI: 10.1103/PhysRevX.2.021006

\section{INTRODUCTION}

Ultrafast magnetization dynamics is one of the hot topics in magnetism since the pioneering experiment performed in 1996 by Beaurepaire and coworkers [1]. After irradiation of a thin nickel film with a sub-100-fs laser pulse, the authors observed a quenching of the sample magnetization proceeding on a time scale well within one picosecond. This observation triggered the field of femtosecond magnetism. On the way to further speed up read-write processes in computer technology, the exploitation of ultrashort optical pulses, triggering magnetization dynamics on femtosecond time scales, is an elegant and promising strategy. Prior to technological application, it is crucial to understand the fundamental physics governing femtosecond magnetism, which has been shown to be a challenging and fascinating task. Indeed, a lot of effort has

\footnotetext{
*cinchett@rhrk.uni-kl.de
}

Published by the American Physical Society under the terms of the Creative Commons Attribution 3.0 License. Further distribution of this work must maintain attribution to the author(s) and the published article's title, journal citation, and DOI.
Subject Areas: Magnetism, Optics

been put into exploring this intriguing phenomenon in the last 15 years, both experimentally and theoretically. Ultrafast demagnetization following laser excitation has been observed in the classical $3 d$ and $4 f$ ferromagnets $(\mathrm{Co}, \mathrm{Fe}, \mathrm{Ni}, \mathrm{Gd}$, and $\mathrm{Tb}$ ), as well as in several alloy systems such as the binary alloys permalloy and CoPt, ferromagnetic materials ( $\mathrm{GdFeCo}$ ), and several half-metals. Different experimental techniques have been implemented in a time-resolved fashion to probe the magnetization dynamics, delivering similar demagnetization times. Such techniques include the magneto-optical Kerr effect (MOKE) [1-5], the magnetic second-harmonic generation $[6,7]$, the X-ray magnetic circular dichroism $[8,9]$, the spin-resolved photoemission [10-12], and the transverse MOKE using a tabletop extreme-ultraviolet source $[13,14]$.

From the theoretical side, essentially three different types of mechanisms have been proposed to play a role in laser-induced ultrafast demagnetization. One type is the redistribution of angular momentum accompanying scattering between various (quasi-)particles, such as electronmagnon [4], electron-electron [15-17], or electron-phonon (e-p) scattering [17-21]. In other models, coherence effects induced by the driving electromagnetic field are 
an essential ingredient for the femtosecond spin dynamics [5,22]. More recently, it was conjectured that, besides local dissipation of spin angular momentum, superdiffusive spin transport may be of relevance [23]. Despite intense experimental and theoretical efforts, there is still no consensus on the relative importance of the different proposed mechanisms. Moreover, there are two prominent and still unsolved controversies: (i) First, some of the models involving scattering between (quasi-)particles assume the optically excited electrons to be in internal thermal equilibrium during the demagnetization process $[4,18,21]$, pointing to the thermal nature of the demagnetization process [17]. This view contrasts with the hypothesis that the main driving mechanism of ultrafast demagnetization would be either a coherent interaction between the laser and the spins $[5,22]$ or superdiffusive (ultrafast) spin transport [23]. (ii) Second, the origin of the two time scales observed in the demagnetization behavior of the $4 f$ ferromagnet $\mathrm{Gd}$ is attributed either to one single microscopic channel involving e-p scattering in the presence of spinorbit interaction [21], or to the existence of two distinct microscopic channels leading, respectively, to an ultrafast demagnetization mediated by the optically excited hotelectron system and to a slower, quasiequilibrium demagnetization process [9].

Here, we present an experimental approach allowing us to uncover the physics behind (i) and (ii). We have studied the dependence of ultrafast demagnetization on the sample ambient temperature ( $\left.T_{\text {ambient }}\right)$ in nickel and compare the experimental results to the theoretical modeling of the spin dynamics accomplished by a generalization of the microscopic three-temperature model (M3TM) [21]. The M3TM is based on the Elliott-Yafet (EY) type of e-p scattering as a microscopic mechanism for modeling ultrafast demagnetization. In order to make the comparison between theory and experiment more stringent, we also consider the fluence dependence of ultrafast demagnetization and model both data sets (dependence on fluence and on $T_{\text {ambient }}$ ) with the same set of material parameters. The effect of $T_{\text {ambient }}$ on ultrafast demagnetization is an especially important issue. After the impact of the femtosecond laser pulse, the electron, phonon, and spin systems are out of equilibrium with respect to each other. By changing the ambient temperature - meaning by creation or annihilation of phonons - the phase space for electron-phonon scattering changes. At the same time, close to the Curie temperature $T_{\mathrm{C}}$, loss of ferromagnetic order and the concomitant reduction of exchange splitting result in a slowing of the magnetization dynamics. Hence, considering ultrafast demagnetization at varying values of $T_{\text {ambient }}$ is a key to identifying the underlying mechanism.

The most striking experimental observation is that, by increasing the ambient temperature, it is possible to drive the ultrafast demagnetization of nickel from a regime where a single exponential decay is observed (termed
Type-I dynamics, typical for $3 d$-ferromagnetic metals at room temperature) to the regime where two time scales for the demagnetization are observed (termed Type-II dynamics, typical for $4 f$-ferromagnetic metals). This observation represents the first experimental evidence that the very same ferromagnetic material can be driven from a Type-I to a Type-II transition by simply changing the ambient temperature. In the following, we will first describe why and under which conditions such transition is expected to occur for nickel. Hereafter, we present the experimental data and analyze it using a significantly refined version of the M3TM introduced in Ref. [21]. A striking resemblance between measurements and the simulations confirms the transition from Type I to Type II at elevated temperatures, emerging from the slower equilibration between the electron and spin system compared to the equilibration between the phonon and spin system. This shows that: (i) Ultrafast demagnetization can be treated theoretically as a thermal process, driven by the difference between the temperature of the electronic system $T_{\mathrm{e}}$ and that of the spin system $T_{\mathrm{s}}$; (ii) The dynamics over the whole temperature and fluence range can be described by a single scattering mechanism.

\section{TYPE-I AND TYPE-II DYNAMICS}

The motivation for the experiments reported in this paper is a prediction made by Koopmans and coworkers in Ref. [21]. This work introduced a quantitative model to describe the magnetization dynamics: the M3TM. Although the model is relatively simple, it reproduces the rich diversity of femtosecond magnetism and addresses the paradoxical difference between the measured demagnetization in the $3 d$ ferromagnets cobalt and nickel as compared to the rare-earth $4 f$-system gadolinium. Basically, the M3TM elucidates that the demagnetization is caused by thermal equilibration between the electron and spin system, microscopically mediated by an EY type of e-p scattering process.

Although only one microscopic scattering mechanism is invoked in the model, two types of magnetization dynamics are naturally reproduced. The first, Type I, occurs when coupling between the electron and spin system is strong. One example of a Type-I trace is depicted in Fig. 1(a).

During the first hundreds of femtoseconds, the magnetization is quenched, whereas a rapid remagnetization occurs during the following picoseconds. Materials exhibiting Type-I behavior in experiments conducted at room temperature are, for example, the $3 d$ ferromagnets. The other type of dynamics, Type II, is present when coupling between the electron and spin system is weak. In this case, a fast initial quenching of the magnetization is followed by a slower decay, i.e., there is a second step visible in the demagnetization. Type-II dynamics are observed in $4 f$ systems such as $\mathrm{Gd}$ and $\mathrm{Tb}$ [9], but also in some halfmetals [24]. 

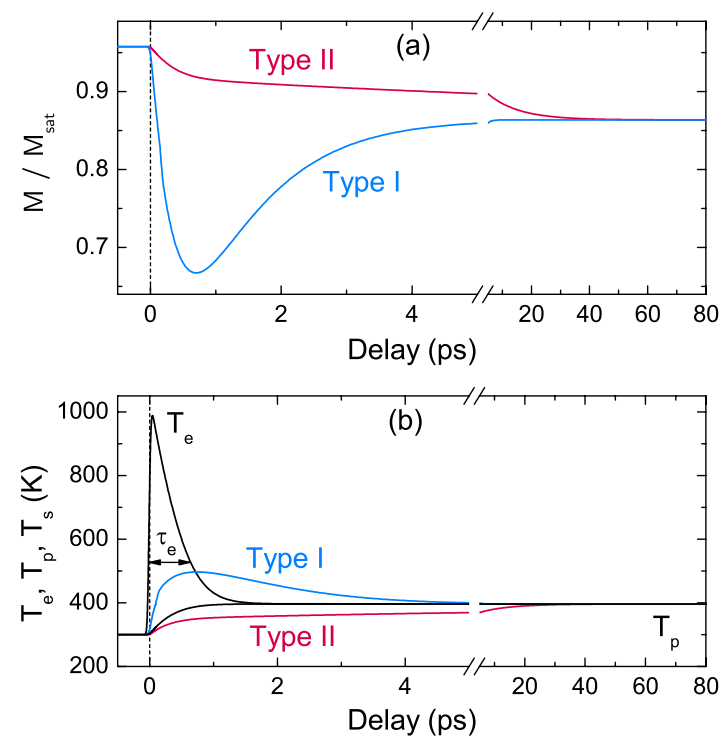

FIG. 1. (a) Examples of Type-I and Type-II magnetization dynamics after excitation by a laser pulse. The magnetization (relative to its value at zero temperature, $M_{\text {sat }}$ ) is plotted as a function of delay time between the pump and probe beams. (b) Temperatures of the electron, phonon, and spin systems ( $T_{\mathrm{e}}, T_{\mathrm{p}}$, and $T_{\mathrm{s}}$, respectively), as a function of time. For the spin system, both Type-I and Type-II traces are depicted.

The origin of the two types of magnetization dynamics is clarified in Fig. 1(b). They are a direct consequence of the equilibration of the electron and spin temperatures. In laser-induced magnetization dynamics, the laser pulse is absorbed by the electron system, typically heating it a few hundred Kelvin. Because of e-p scattering, the electron and phonon systems equilibrate within a time $\tau_{\mathrm{e}}$, which is usually approximately $1 \mathrm{ps}$.

For the case of Type-I dynamics, coupling to the electron system is strong, and the spin system is heated within just a few hundred femtoseconds, i.e., during the time when the electron temperature $T_{\mathrm{e}}$ is large. After approximately $0.5-1 \mathrm{ps}, T_{\mathrm{e}}$ becomes smaller than the spin temperature $T_{\mathrm{s}}$ [Fig. 1(b), blue solid line], which is caused by the cooling of the electron system due to equilibration with the phonons. This cooling leads to a remagnetization, which is less efficient than the demagnetization due to the smaller difference between $T_{\mathrm{e}}$ and $T_{\mathrm{s}}$.

For weak coupling, the precondition for Type-II dynamics, a fast demagnetization also occurs during the first hundreds of femtoseconds. However, $T_{\mathrm{s}}$ never exceeds the phonon temperature $T_{\mathrm{p}}$ [Fig. 1(b), magenta solid line], as the coupling of the spin system to the electron system is not efficient enough. After e-p equilibration, the spin system is still relatively "cool" with respect to the electron and phonon systems, and demagnetization proceeds for a longer time. Hence, the two time scales observed for the two demagnetization steps, and characteristic for a Type-II dynamics, are determined solely by the difference between $T_{\mathrm{e}}$ and $T_{\mathrm{s}}$. When the electron system is hot, $T_{\mathrm{e}}-T_{\mathrm{s}}$ is large, while, after e-p equilibration, this difference is considerably smaller, leading to a long and slow demagnetization. Experimentally verifying the transition between Type-I and Type- II behavior in the same material system would give strong evidence that the ultrafast dynamics are governed just by the thermalization of the electron and the spin systems. In addition, contrary to what is claimed for the $4 f$ ferromagnets $\mathrm{Gd}$ and $\mathrm{Tb}$ [9], the transition would show that only one single scattering mechanism is at the origin of both steps in the demagnetization, where the rate of demagnetization is merely determined by the temperature difference between $T_{\mathrm{e}}$ and $T_{\mathrm{s}}$.

The M3TM predicts Type-II behavior when at least one of the following conditions is satisfied: (i) The ferromagnet has a large magnetic moment, (ii) The microscopic scattering mechanism - coupling the electron and spin system - is weak, or (iii) The experiments are conducted close to $T_{\mathrm{C}}$. In Ref. [21], Koopmans and coworkers predicted that a transition from Type-I to Type-II behavior should occur in nickel by increasing the ambient temperature to close to $T_{\mathrm{C}}$, i.e., when satisfying condition (iii). The reason for such transition is that, close to $T_{\mathrm{C}}$, loss of ferromagnetic order results in a weaker exchange splitting $\Delta_{\text {ex }}$. Since in the M3TM the rate of demagnetization depends on $\Delta_{\text {ex }}$ (introduced self-consistently in the model as a function of the magnetization itself), performing the experiments close to $T_{\mathrm{C}}$ inevitably leads to a slower demagnetization rate. As a result, the spin temperature $T_{\mathrm{s}}$ proceeds much slower at elevated ambient temperatures, leading to Type-II behavior.

\section{METHODS}

To investigate whether the predicted transition from Type I to Type II is indeed present, we perform a comprehensive experimental exploration of laser-induced magnetization dynamics in nickel, spanning a large parameter space. We undertake measurements on the de- and remagnetization dynamics by means of the time-resolved magneto-optical Kerr effect (TR-MOKE) for a wide range of fluences $\left(2.5-5.0 \mathrm{~mJ} / \mathrm{cm}^{2}\right)$ and ambient temperatures $T_{\text {ambient }}(80-480 \mathrm{~K})$. In order to examine the temperaturedependent spin dynamics, we implemented a cold-finger cryostat (MicrostatHe, Oxford Instruments), with the temperature controllable over a range from $77-500 \mathrm{~K}$, in a longitudinal Kerr geometry [25]. For the measurement we utilized a bichromatic pump-probe scheme (pump at $800 \mathrm{~nm}$, probe at $400 \mathrm{~nm}$ ) to minimize optical artifacts like dichroic bleaching [3]. The laser pulses were generated by a multipass amplifier system running at a repetition rate of $1 \mathrm{kHz}$ and possessing a FWHM of approximately $50 \mathrm{fs}$ at the sample position. A beam stabilization guaranteed the spatial pump-probe overlap during the measurements. The sample under investigation is an in-plane magnetized, polycrystalline, 15 -nm-thin nickel film that was fabricated by electron-beam evaporation on an insulating substrate (oxidized Si surface). Fifteen $\mathrm{nm}$ is on the order of the 
penetration depth of the pump-laser wavelength. Hence, in a first order approach, the whole Ni film is heated by the pump laser and hot-electron transport out of the investigated region is minimized $[23,26]$.

To analyze the data, we used a refined version of the M3TM because the equations that were derived in Ref. [21] are valid only when $T_{\text {ambient }}>T_{\text {Debye }}$, where $T_{\text {Debye }}$ is the Debye temperature. Furthermore, the spinspecific heat is neglected in Ref. [21], which is not a valid assumption close to $T_{\mathrm{C}}$. For the model used to analyze the presented data, both restrictions are dropped. The detailed theoretical background and analysis of the generalized model will be elaborated in a future work [27].

\section{MEASUREMENTS AND INTERPRETATION}

The effect of the external excitation scheme on femtosecond spin dynamics is studied by observing the dependence on the fluence of the pump pulse (referred to as fluence scenario) as well as on $T_{\text {ambient }}$ (temperature scenario) (see Figs. 2 and 3, respectively). In the fluence scenario, the sample is at room temperature, and the fluence of the pump pulse (source term) $P_{0}$ is varied from $2.5-5.0 \mathrm{~mJ} / \mathrm{cm}^{2}$ in steps of $0.5 \mathrm{~mJ} / \mathrm{cm}^{2}$. The evolution of the transient magnetization $M$, as normalized to the saturation magnetization $M_{0}$ before the arrival of the pump pulse, is displayed in Fig. 2. In the temperature scenario (Fig. 3), the ambient temperature $T_{\text {ambient }}$ of the system (initial equilibrium temperature) is changed from 80 to $480 \mathrm{~K}$, while keeping the fluence of the pump pulse at a constant level of $3.5 \mathrm{~mJ} / \mathrm{cm}^{2}$.

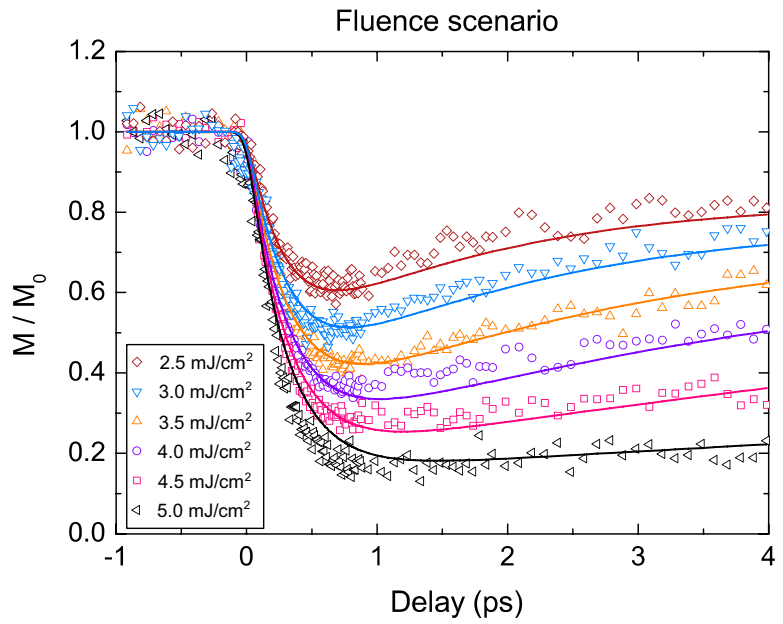

FIG. 2. Fluence dependence of the laser-induced magnetization dynamics of nickel at room temperature $\left(T_{\text {ambient }}=300 \mathrm{~K}\right)$. The fluence is adjusted to six levels of the pump pulse ranging from $2.5-5.0 \mathrm{~mJ} / \mathrm{cm}^{2}$ in steps of $0.5 \mathrm{~mJ} / \mathrm{cm}^{2}$. (Symbols correspond to measurements.) The magnetization is normalized to its value at negative time delays. Lines are calculations with the refined M3TM, using the microscopic parameters obtained by fitting the $3.5 \mathrm{~mJ} / \mathrm{cm}^{2}$ measurement.

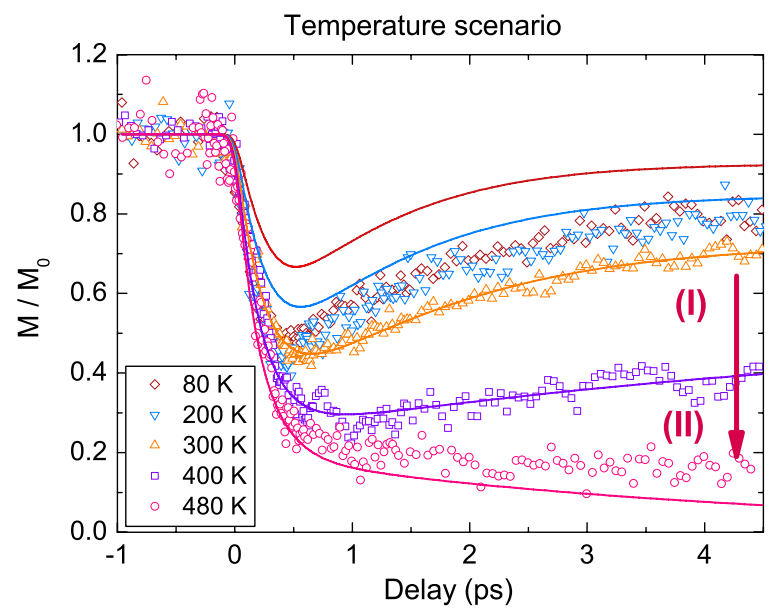

FIG. 3. Dependence of the ultrafast demagnetization of nickel on the ambient temperature $T_{\text {ambient }}$ at a fluence of $P_{0}=$ $3.5 \mathrm{~mJ} / \mathrm{cm}^{2}$. Demagnetization dynamics measured for five different ambient temperatures from 80-480 K (symbols). Lines are calculations with the refined M3TM, using the microscopic parameters obtained by fitting the $300 \mathrm{~K}$ measurement.

The fluence scenario in Fig. 2 exhibits a pronounced increase of the maximum magnetization quenching as a function of fluence, as well as a slowing of both the deand remagnetization. We conclude that the maximum quenching follows an approximately linear relation to the pump fluence, as has been reported previously [6,28]. Qualitatively, the temperature scenario in Fig. 3 reveals a similar behavior, but two striking features appear. First, even at constant laser fluence, increasing the ambient temperature leads to a considerably larger maximum quenching. Second, and more important, at $480 \mathrm{~K}$ the predicted transition from one-step (Type-I) to two-step (Type-II) dynamics occurs. The double-exponential behavior, with time scales of approximately 0.2 and 4 ps, respectively, is also highlighted in Fig. 4(a).
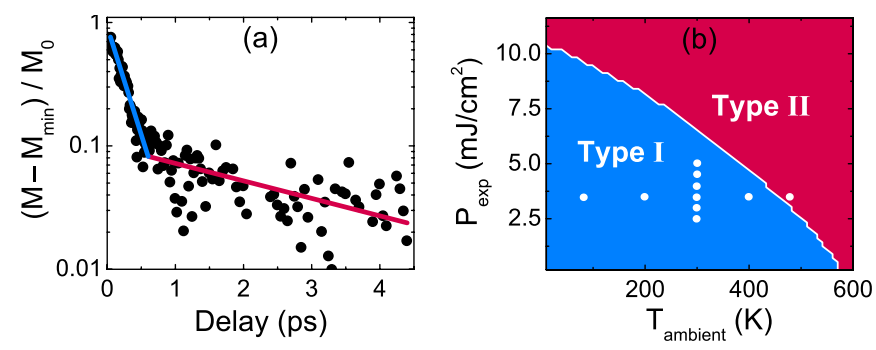

FIG. 4. Two-step dynamics of nickel. (a) Experimental verification of two-step dynamics in nickel at an ambient temperature of $480 \mathrm{~K}$. (The data are taken from Fig. 3.) $M_{\min }$ denotes the minimum magnetization, and the lines serve as a guide to the eye. For the fast step, we obtain a time constant of approximately $0.2 \mathrm{ps}$, whereas the slow step occurs within approximately 4 ps. (b) Calculated phase diagram for ultrafast demagnetization in nickel. The white dots indicate the operating conditions of the experiments in Figs. 2 and 3. 
To show that measured magnetization dynamics can be qualitatively explained by scattering due to a single microscopic mechanism, we apply the refined M3TM. To analyze the data, the trace at $300 \mathrm{~K}$ and $3.5 \mathrm{~mJ} / \mathrm{cm}^{2}$ is fitted with the M3TM, where the fluence, the e-p coupling constant $\lambda_{\mathrm{ep}}$, and the spin-flip probability $a_{\mathrm{sf}}$ are fit parameters. For the material parameters of nickel, the same values have been used as in Ref. [21]. Only the heat capacity of the electron system has been readdressed, using the values measured by Meschter et al. [29], because the previous value significantly deviated from those obtained by most caloritronic measurements.

The fits yield $\lambda_{\mathrm{ep}}=23 \mathrm{meV}$ and $a_{\mathrm{sf}}=0.08$. This means that the obtained spin-flip parameter $\left(a_{\mathrm{sf}}\right)$ for nickel is approximately 2 times smaller than earlier reported [21], which is due mainly to the different heat capacity of the electron system. This large change of $a_{\mathrm{sf}}$ shows that the exact spin-flip parameter is difficult to obtain from experiments, as it is very sensitive to the other material parameters. On the other hand, both values obtained for $a_{\mathrm{sf}}$ lie well within the range formerly obtained by ab-initio calculations [20]. Moreover, $a_{\text {sf }}$ fits well to very recent evaluations for nickel, which include the specific band structure, the ab-initio-calculated phonon spectrum, and the full Eliashberg function for e-p coupling [30]. We note that Refs. $[30,31]$ argue convincingly that the EY process in a ground-state band structure is not efficient enough to reproduce the measured demagnetization. Our present analysis goes beyond a ground-state band structure because it implicitly contains a dynamical change of the magnetic structure, which is modeled by a phenomenological spin temperature for a quasiequilibrium situation.

We use a virtually identical set of material parameters ( $\lambda_{\mathrm{ep}}=26 \mathrm{meV}, a_{\mathrm{sf}}=0.08$ ) to predict the temperature dependence of the demagnetization of nickel. The predictions for both the fluence scenario and the temperature scenario are plotted as solid lines in Figs. 2 and 3, respectively. In the fluence scenario, the calculated traces reproduce the measurements very well. Both the increase in the maximum quenching and the altered remagnetization are accurately reproduced on increasing the fluence in the calculations. This demonstrates that indeed a single scattering mechanism can explain the measurements in the fluence scenario. However, small deviations between the calculations and experiments visible, for example, for the largest fluence, indicate that either the model is oversimplified or that other mechanisms, like electron-electron scattering [15] or superdiffusive spin transport [23], also contribute to the demagnetization.

In the temperature scenario, deviations between the measurements and calculations are more pronounced, mainly for $T_{\text {ambient }}<300 \mathrm{~K}$. Although the shape of the demagnetization traces is accurately reproduced, the maximum quenching is underestimated in the calculations. Again, simplifications of the model such as the assumption of an idealized electron and phonon band structure could be the reason. What we want to stress, however, is that, qualitatively, the model mirrors the measurements accurately. Importantly, the transition from Type-I to Type-II behavior is reproduced, and the calculated transition temperature corresponds to the one observed in the experiment. To illustrate this, a phase diagram of nickel is constructed with the refined M3TM and depicted in Fig. 4(b). The white dots in the phase diagram correspond to the experimental conditions for the traces in Figs. 2 and 3, where the color regions clarify that increasing either the fluence or the ambient temperature would induce a transition from Type I to Type II. Note, however, that the maximum fluence of $5.0 \mathrm{~mJ} / \mathrm{cm}^{2}$ as utilized in the experiments presented in Fig. 2 is both experimentally and theoretically insufficient to cause such a transition at room temperature.

The second step in the demagnetization of nickel, as predicted by the refined M3TM, indicates that one single microscopic mechanism, which couples the spin temperature to the electron temperature, can fully explain the magnetization dynamics on all time scales in the $3 d$ ferromagnets. The suggestion that spin-lattice relaxation dominates during the second step, as has been claimed for $\mathrm{Gd}$ and $\mathrm{Tb}$ [9], can be ruled out, since spin-lattice relaxation has been estimated to be approximately $300 \mathrm{ps}$ [32] in nickel. The fact that both one-step and two-step dynamics are now found for the very same material makes it much more plausible that there is only one main mechanism responsible for the two steps. The observed slowing near $T_{\mathrm{C}}$ strongly suggests that the thermalizing spin system plays a large role in the magnetization dynamics. It has recently been shown that femtosecond demagnetization and picosecond-nanosecond damping have the same origin in $\mathrm{Ni}, \mathrm{Co}$, and $\mathrm{Gd}[33]$ and can be modeled on the same footing, namely, by thermal coupling of the electron system to the spin system. Although it is surprising that the same spin dynamics hold on both time scales, our measurements support these findings.

\section{CONCLUSION}

We have studied the dependence of ultrafast demagnetization in nickel on the fluence of the exciting laser and on the sample ambient temperature $T_{\text {ambient }}$. The behavior of the demagnetization dynamics in both the fluence and the temperature scenarios-including the transition from Type-I to Type-II dynamics at sufficiently high $T_{\text {ambient }}-$ can be reproduced by the refined M3TM by using the same set of material parameters. This solves two current controversies: (i) Ultrafast demagnetization can be treated theoretically as a thermal process, driven by the difference between the temperatures of the electronic system $T_{\mathrm{e}}$ and the spin system $T_{\mathrm{s}}$; (ii) The demagnetization dynamics over the whole fluence and temperature range-including the presence of two time scales in the demagnetization 
traces_-can be described by invoking a single scattering mechanism.

\section{ACKNOWLEDGMENTS}

We thank Manfred Fähnle, Grégory Malinowski, and Peter Oppeneer for fruitful discussions. The DFG within the GRK 792 is acknowledged for financial support. This work is part of the research program of the Foundation for Fundamental Research on Matter (FOM), which is part of the Netherlands Organisation for Scientific Research (NWO).

[1] E. Beaurepaire, J.-C. Merle, A. Daunois, and J.-Y. Bigot, Ultrafast Spin Dynamics in Ferromagnetic Nickel, Phys. Rev. Lett. 76, 4250 (1996).

[2] J. Güdde, U. Conrad, V. Jähnke, J. Hohlfeld, and E. Matthias, Magnetization Dynamics of Ni and Co Films on $\mathrm{Cu}(001)$ and of Bulk Nickel Surfaces, Phys. Rev. B 59, R6608 (1999).

[3] B. Koopmans, M. van Kampen, J. T. Kohlhepp, and W. J. M. de Jonge, Ultrafast Magneto-Optics in Nickel: Magnetism or Optics?, Phys. Rev. Lett. 85, 844 (2000).

[4] E. Carpene, E. Mancini, C. Dallera, M. Brenna, E. Puppin, and S. De Silvestri, Dynamics of Electron-Magnon Interaction and Ultrafast Demagnetization in Thin Iron Films, Phys. Rev. B 78, 174422 (2008).

[5] J.-Y. Bigot, M. Vomir, and E. Beaurepaire, Coherent Ultrafast Magnetism Induced by Femtosecond Laser Pulses, Nature Phys. 5, 515 (2009).

[6] J. Hohlfeld, E. Matthias, R. Knorren, and K.H. Bennemann, Nonequilibrium Magnetization Dynamics of Nickel, Phys. Rev. Lett. 78, 4861 (1997).

[7] M. Sultan, A. Melnikov, and U. Bovensiepen, Ultrafast Magnetization Dynamics of Gd(0001): Bulk versus Surface, Phys. Status Solidi B 248, 2323 (2011).

[8] C. Stamm, T. Kachel, N. Pontius, R. Mitzner, T. Quast, K. Holldack, S. Khan, C. Lupulescu, E. F. Aziz, M. Wietstruk, H. A. Durr, and W. Eberhardt, Femtosecond Modification of Electron Localization and Transfer of Angular Momentum in Nickel, Nature Mater. 6, 740 (2007).

[9] M. Wietstruk, A. Melnikov, C. Stamm, T. Kachel, N. Pontius, M. Sultan, C. Gahl, M. Weinelt, H. A. Dürr, and U. Bovensiepen, Hot-Electron-Driven Enhancement of Spin-Lattice Coupling in $G d$ and $T b$ of Ferromagnets Observed by Femtosecond X-Ray Magnetic Circular Dichroism, Phys. Rev. Lett. 106, 127401 (2011).

[10] H.-S. Rhie, H. A. Dürr, and W. Eberhardt, Femtosecond Electron and Spin Dynamics in Ni/W(110) Films, Phys. Rev. Lett. 90, 247201 (2003).

[11] A. B. Schmidt, M. Pickel, M. Donath, P. Buczek, A. Ernst, V.P. Zhukov, P. M. Echenique, L. M. Sandratskii, E. V. Chulkov, and M. Weinelt, Ultrafast Magnon Generation in an Fe Film on Cu(100), Phys. Rev. Lett. 105, 197401 (2010).

[12] A. Goris, K. M. Döbrich, I. Panzer, A. B. Schmidt, M. Donath, and M. Weinelt, Role of Spin-Flip Exchange
Scattering for Hot-Electron Lifetimes in Cobalt, Phys. Rev. Lett. 107, 026601 (2011).

[13] C. La-O-Vorakiat, M. Siemens, M. M. Murnane, H. C. Kapteyn, S. Mathias, M. Aeschlimann, P. Grychtol, R. Adam, C. M. Schneider, J. M. Shaw, H. Nembach, and T. J. Silva, Ultrafast Demagnetization Dynamics at the $m$ Edges of Magnetic Elements Observed Using a TableTop High-Harmonic Soft X-Ray Source, Phys. Rev. Lett. 103, 257402 (2009).

[14] C. La-O-Vorakiat, E. Turgut, C. A. Teale, H. C. Kapteyn, M. M. Murnane, S. Mathias, M. Aeschlimann, C. M. Schneider, J. M. Shaw, H. T. Nembach, and T. J. Silva, Ultrafast Demagnetization Measurements Using Extreme Ultraviolet Light: Comparison of Electronic and Magnetic Contributions, Phys. Rev. X 2, 011005 (2012).

[15] M. Krauß, T. Roth, S. Alebrand, D. Steil, M. Cinchetti, M. Aeschlimann, and H.C. Schneider, Ultrafast Demagnetization of Ferromagnetic Transition Metals: The Role of the Coulomb Interaction, Phys. Rev. B 80, 180407 (2009).

[16] D. Steil, S. Alebrand, T. Roth, M. Krauß, T. Kubota, M. Oogane, Y. Ando, H. C. Schneider, M. Aeschlimann, and M. Cinchetti, Band-Structure-Dependent Demagnetization in the Heusler Alloy $\mathrm{Co}_{2} \mathrm{Mn}_{1-x} \mathrm{Fe}_{x} \mathrm{Si}$, Phys. Rev. Lett. 105, 217202 (2010).

[17] B. Y. Mueller, T. Roth, M. Cinchetti, M. Aeschlimann, and B. Rethfeld, Driving Force of Ultrafast Magnetization Dynamics, New J. Phys. 13, 123010 (2011).

[18] B. Koopmans, J. J.M. Ruigrok, F. Dalla Longa, and W.J.M. de Jonge, Unifying Ultrafast Magnetization Dynamics, Phys. Rev. Lett. 95, 267207 (2005).

[19] M. Cinchetti, M. Sanchez Albaneda, D. Hoffmann, T. Roth, J.-P. Wustenberg, M. Krauss, O. Andreyev, H. C. Schneider, M. Bauer, and M. Aeschlimann, Spin-Flip Processes and Ultrafast Magnetization Dynamics in Co: Unifying the Microscopic and Macroscopic View of Femtosecond Magnetism, Phys. Rev. Lett. 97, 177201 (2006).

[20] D. Steiauf and M. Fähnle, Elliott-Yafet Mechanism and the Discussion of Femtosecond Magnetization Dynamics, Phys. Rev. B 79, 140401 (2009).

[21] B. Koopmans, G. Malinowski, F. Dalla Longa, D. Steiauf, M. Fähnle, T. Roth, M. Cinchetti, and M. Aeschlimann, Explaining the Paradoxical Diversity of Ultrafast LaserInduced Demagnetization, Nature Mater. 9, 259 (2010).

[22] G.P. Zhang and W. Hübner, Laser-Induced Ultrafast Demagnetization in Ferromagnetic Metals, Phys. Rev. Lett. 85, 3025 (2000).

[23] M. Battiato, K. Carva, and P. M. Oppeneer, Superdiffusive Spin Transport as a Mechanism of Ultrafast Demagnetization, Phys. Rev. Lett. 105, 027203 (2010).

[24] G. M. Müller, J. Walowski, M. Djordjevic, G.-X. Miao, A. Gupta, A. V. Ramos, K. Gehrke, V. Moshnyaga, K. Samwer, J. Schmalhorst, A. Thomas, A. Hütten, G. Reiss, J.S. Moodera, and M. Münzenberg, Spin Polarization in Half-Metals Probed by Femtosecond Spin Excitation, Nature Mater. 8, 56 (2008).

[25] T. Roth, D. Steil, D. Hoffmann, M. Bauer, M. Cinchetti, and M. Aeschlimann, Dynamics of the Coercivity in Ultrafast Pump-Probe Experiments, J. Phys. D 41, 164001 (2008). 
[26] M. Aeschlimann, M. Bauer, S. Pawlik, R. Knorren, G. Bouzerar, and K. H. Bennemann, Transport and Dynamics of Optically Excited Electrons in Metals, Appl. Phys. A 71, 485 (2000).

[27] A. J. Schellekens, T. Roth, M. Cinchetti, M. Aeschlimann, and B. Koopmans (to be published).

[28] U. Atxitia, O. Chubykalo-Fesenko, J. Walowski, A. Mann, and M. Münzenberg, Evidence for Thermal Mechanisms in Laser-Induced Femtosecond Spin Dynamics, Phys. Rev. B 81, 174401 (2010).

[29] P. J. Meschter, J. W. Wright, C. R. Brooks, and T. G. Kollie, Physical Contributions to the Heat Capacity of Nickel, J. Phys. Chem. Solids 42, 861 (1981).

[30] K. Carva, M. Battiato, and P. M. Oppeneer, Ab Initio Investigation of the Elliott-Yafet Electron-Phonon
Mechanism in Laser-Induced Ultrafast Demagnetization, Phys. Rev. Lett. 107, 207201 (2011).

[31] S. Essert and H. C. Schneider, Electron-Phonon Scattering Dynamics in Ferromagnetic Metals and Their Influence on Ultrafast Demagnetization Processes, Phys. Rev. B 84, 224405 (2011).

[32] G. Zhang, W. Hübner, E. Beaurepaire, and J.-Y. Bigot, in Spin Dynamics in Confined Magnetic Structures I, Topics in Applied Physics Vol. 83, edited by B. Hillebrands and K. Ounadjela (Springer-Verlag, Berlin, 2002), pp. 245289, ISBN 10.1007/3-540-40907-6_8.

[33] U. Atxitia and O. Chubykalo-Fesenko, Ultrafast Magnetization Dynamics Rates within the LandauLifshitz-Bloch Model, Phys. Rev. B 84, 144414 (2011). 